\title{
Case Report: Measles in a 23-Day-0ld Newborn
}

\author{
Jacqueline Stephanie Fernandes do Nascimento ${ }^{1}$, Janie Kelly Fernandes do Nascimento ${ }^{2}$, Marco \\ Antônio Alves Aziz ${ }^{3}$, Dóris Augusta Caldas Rebello, Maria Angélica Barcellos Svaiter ${ }^{3}$, Carlos \\ Henrique Melo Reis ${ }^{4}$, Carlos Eduardo Cardoso ${ }^{5}$, Guilherme Gomes Azizi ${ }^{6}$, Brian França dos \\ Santos $^{3}$, Mariana Barcellos Svaiter ${ }^{3}$, Adalgiza Mafra Moreno ${ }^{3}$, Marco Antonio Araújo Leite ${ }^{7}$, Nilson \\ Gomes $^{8}$ and Marco Antônio Orsini Neves ${ }^{9}$
}

${ }^{1}$ Medicine Department, Iguaçu University, Brazil

${ }^{2} U N I G$, Iguaçu University, Brazil

${ }^{3}$ Faculty of Medicine, Iguaçu University, Brazil

${ }^{4}$ Iguaçu University - UNIG and Nova Iguaçu General Hospital, Brazil

${ }^{5}$ University of Vassouras, Brazil

${ }^{6}$ Department of Immunology, Federal University of Rio de Janeiro, Brazil

${ }^{7}$ Fluminense Federal University - UFF, Brazil

${ }^{8}$ Iguaçu University, UNIG - Faculty of Medicine and Director of Maternidade Mariana Bulhões, Brazil

${ }^{9}$ Member of the Neurology and Neurosurgery Service at UFF, Iguaçu University, Brazil

*Corresponding author: Jacqueline Stephanie Fernandes do Nascimento, Rua Débora de Souza Costa, Nova Iguaçu, Rio de Janeiro, Brazil.

To Cite This Article: Jacqueline Stephanie Fernandes do N, Janie Kelly Fernandes do N, Marco Antônio Alves A, Dóris Augusta Caldas R, Carlos Eduardo C, et al., Case Report: Measles in a 23-Day-Old Newborn. 2020 - 8(5). AJBSR.MS.ID.001317. DOI: 10.34297/AJBSR.2020.08.001317.

Received: 眥 April 28, 2020; Published: 笽 May 04, 2020

\begin{abstract}
For many years Measles has been shown to be one of the main reasons for childhood mortality and morbidity, especially in the age group below 1 year old. Although rare, there are reports in the literature that corroborate infections in newborns. This article aimed to evaluate and expose, through a case report, the clinical course of Measles diagnosed in a 23-day-old newborn, in addition to describing, through a bibliographic search, the history of the triple viral vaccine and recommended indications for the use of polyvalent immunoglobulin. It is concluded that Measles is considered rare in newborns, although it is possible to affect them in cases of susceptible mothers, and therefore further studies are needed in favor of clinical knowledge of the disease course in this age group.
\end{abstract}

Keywords: Vaccine; measles; newborn; Immunoglobulin

\section{Introduction}

Established as a viral disease, highly contagious and acute infectious, Measles is characterized by being a febrile exanthematous disease, caused by viruses of the family Paramyxoviridae, of the genus Morbillivirus, which has eight classes and 24 genotypes and the human being is its only reservoir and source of infection. Its classic affection is in childhood, with no preference for race or gender and with global distribution [1,2,3].
Measles is transmitted through contact with nasopharynx droplets that are dispersed in the air the moment the individual speaks, coughs, breathes or sneezes, or sporadically by aerosol in the upper airway mucosa or conjunctiva [4]. The patient, when infected, can transmit Measles for 6 days before the onset of therashes and up to 4 days after their disappearance, the period most likely to be transmitted is 2 days before and 2 days after the onset of the rashes [1,5]. 
The classic clinical condition is associated with fever, coryza, conjunctivitis, photophobia, morbilliform maculopapular rash, with cephalocaudal distribution, that is, it starts on the forehead, around the ears and neck, in the hairline and in the cranio-caudal direction, associated respiratory symptoms, such as dry cough. It presents tropism in the upper airways and can evolve with serious complications, such as otitis, laryngitis, diarrhea, in addition to pneumonia and encephalitis, which are the main causes responsible for deaths $[1,5]$.

Symptoms appear 10-12 days after the infection. The incubation period, which goes from the moment of infection to the appearance of the first symptom, is on average 10 days (7-21) [1,5]. Its transmission occurs from the initial phase of the symptoms until the convalescence period. An unfavorable socioeconomic situation is closely related to a greater lethality of Measles. Faced with an arsenal of non-immunized individuals, the virus, which is very contagious, can be preserved at endemic levels, with a seasonal character, causing regular epidemics [5].

Since 1975, Measles has been a compulsory notification disease. Any case considered suspicious, in other words, that shows typical signs and symptoms of the disease, such as: fever, rash, dry cough, coryza or conjunctivitis, regardless of the vaccination situation or age group, must be notified immediately to health surveillance within 24 hours [5]. The virus located in the circulating blood, resulting from the infection, causes disseminated vasculitis, which causes clinical manifestations [7].

Responsible for being one of the major causes of hospitalization and morbidity in children under 5 years of age, mainly in malnourished and in those in developing countries. Lethality is associated with socioeconomic status, as well as healthcare, hygiene and nutrition $[1,6]$. Due to the transplacental transfer of maternal antibodies, the disease is considered rare in newborns, although it is possible to affect them in cases of susceptible mothers.

\section{Case Report}

Female newborn, 23 days old, presenting 1 day ago, rhinorrhea and morbilliform maculopapular rash on the face, trunk and upper and lower limbs (Figure 1), associated with whitish macules in the oral cavity suggestive of Koplik's spots (Figure 2). Her guardian denies other symptoms involved in the newborn, nor previous pathologies and use of regular medications. The mother also reports having been affected by a recent manifestation of maculopapular cutaneous rash on the trunk and upper and lower limbs.
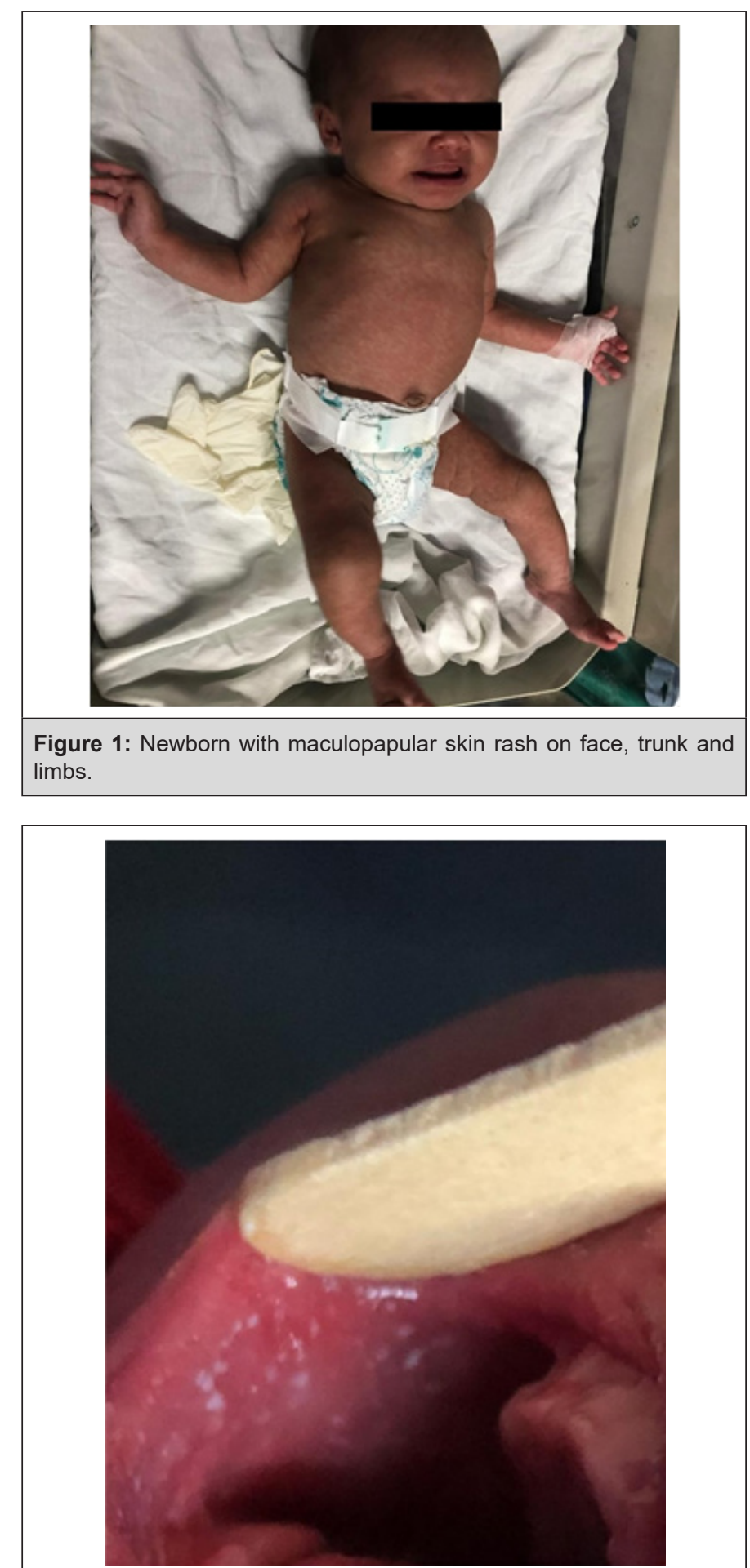

Figure 2: Whitish spots in the oral cavity suggestive of Koplik's sign.

Vaccine history of BCG and Hepatitis B administered in the delivery room after birth. Food history of exclusive breastfeeding. 
Family history without comorbidities. In social history, patient lives with his parents and sister, in a masonry house, benefiting from basic sanitation and piped water. Child development history showed normal neuro-psychomotor evolve for the age.

The clinical examination of the child showed a patient in good general condition, active and reactive, hydrated, anicteric, acyanotic, eupneic and eucardic in environmental air. Presence of morbilliform maculopapular rash on the face, trunk and limbs; and whitish macules in the oral cavity. Cardiovascular system at regular heart rhythm, normophonetic sounds in two times, without murmurs or extrasystoles. Respiratory system showed a universally audible vesicular murmur, without adventitious sounds. The abdomen was flaccid, peristaltic, painless on superficial and deep palpation, and no signs of visceromegaly. Lower limbs showed no edema and peripheral pulses present.

The child was admitted to the ward, with respiratory isolation, from a hospital in the state of Rio de Janeiro; and the following tests were requested: FullBlood Count, IgG and IgM serologies and RT-PCR for measles. The examination of serology for measles was positive for both IgG and IgM and the RT-PCR was positive for the virus as well. The newborn was hospitalized for 3 days and was offered support and symptomatic drugs, such as analgesics and antiemetics during her hospitalization, her vital signs were monitoredeach 6 hours. The patient did not develop fever or other complications and was in good general condition. She was discharged due to clinical improvement.

\section{Discussion}

Since the development and widespread use of the measles vaccine, epidemics have become uncommon8, however, this has not always been the case. For many years, Measles has been shown to be one of the main reasons for childhood mortality and morbidity, especially in the age group below 1 year old. In 1960, measles vaccine was introduced in Brazil, and its use in public health was due to the work of state governments that imported the immunobiological component of the international market, even if in a discontinuous way [9].

The National Immunization Program - PNI, was developed in 1973 , with the primary aim of promoting, organizing and judging immunization actions across the country. In this year and in 1974, vaccination campaigns were carried out in several urban areas of the state, and in the early 1980s, areas with less vaccination coverage were contemplated. This tactic was immediately replaced by basic health services and recognition of routine services. In 1987 and 1988, mass vaccination campaigns against Measles were promoted in the state of São Paulo and Paraná, respectively, in favor of the control and eradication of the disease [9].

Aiming to achieve the disease elimination, in 1992, Brazil instituted the National Measles Elimination Plan as the main goal of its health policy, being the first national vaccination campaign against the disease [9]. The country received, in 2016, the certificate of Measles eradication by the World Health Organization (WHO), publicly decreeing measles-free territory for the Americas. It was observed that there was a decline in vaccination coverage, about 10 to $20 \%$, as of the year 2016. Based on this information, the option of not vaccinating and manipulating individuals in their circle of coexistence, not to do as well, collaborates to decrease the herd immunity, being able to originate localized outbreaks or the alarming increase of contamination in specific groups or people [10].

Therefore, it is possible to affirm that there is an inversely proportional relation between the exponential increase in the incidence of Measles and the decrease in vaccination coverage, seen especially since 2014. It is evident that vaccine uncertainty is shown as a current propensity not only from the Brazilian population, but as in other nationalities, cooperating to increase epidemics of vaccine-preventable diseases, such as Measles [10].

The World Health Organization (WHO) included in its report, the anti-vaccine movement as a major threat to global health. Considering that children under 1 year of age receive protection from herd immunity, resulting from the mass vaccination of the population, since the age range for vaccination of the first dose is at 12 months, reinforced at 15 months, it does not contemplate newborns. According to the WHO, this movement is deleterious because it puts at risk the advances achieved in the fight against vaccine-preventable diseases, such as Polio and Measles, in addition to rendering herd immunity ineffective [11].

There is a lot of discussion about the reasons why people decide not to vaccinate, among them: they claim that vaccines are inefficient and therefore not safe because they cause adverse effects, they indicate religious reasons, they claim to develop autism in children beyond the very short vaccination interval. There is another aspect that defends the use of the vaccine as a form of population control used by the government [11]. Studies show that the occurrence of Measles during pregnancy before the implementation of the vaccine was approximately 0.5 cases per 10,000 pregnancies. Concluding that, certainly, herd immunity was responsible for the exponential decrease in cases from the introduction of the vaccine [12].

Measles are rare during pregnancy and especially during labor, and the viral effect on the mother and fetus is not well understood. It is known that if the mother was not infected with Measles, there will be no transmission of immunity to the child. In childhood, infection is not recurrent in very young babies, but this rule of immunity is not guaranteed, and there may be a difference between individuals [12]. If Measles occurs before the eighth month of pregnancy, the possibility of prematurityand spontaneous abortion is high, usually occurring at the height of the rash eruption [13]. The implications for the fetus will depend on the stage of pregnancy, 
they are: prematurity, abortions and intrauterine death.

The lack of reported cases and of any common pattern of abnormalities, especially when considering the frequency of previously reported epidemics, suggests that there is no relation with infection and fetal malformations. Some studies on the disease have failed to try to associate a teratogenic effect. Five mothers were described in bibliographies whose average period between the onset of the disease and childbirth lasted 3.5 days. All were preterm babies and one was stillborn with malformations. There are reports of epidemics that, in general, do not attribute maternal infection to congenital malformations; however, prematurity and abortion have a higher incidence in infected mothers [12]. The handling of Measles during pregnancy is limited to symptomatic patients only, but fetal surveillance should be included according to the stages of pregnancy. Pregnant women are only admitted to the hospital when severe symptoms are present, with respiratory isolation to prevent hospital transmission [14].

Hematogenous transplacental transmission is well documented and congenital measles is characterized by the presence of rash at birth and infection acquired in the uterus, with of rashes eruptions present in the tenth day of life. Maternal measles immediately before delivery, in non-immunized pregnant women, constantly involves the fetus. Congenital measles is rare, with its most prevalent reports in the 50s and 60s. The appearance of the disease in newborn varies from mild to cases in which the skin rash is temporary, not always showing Koplik's spots, rapidly fatal types [15].

It was found that when a mother acquires Measles before pregnancy, her child usually has immunity for at least the first five months of life. This immunity is transmitted by placental transfer [13]. It is possible that the infection in newborns and very young children is greatly modified by maternal IgG antibodies and that the disease takes place with a totally atypical course, with very few characteristic signs, which can lead to abortion, especially in preterm, pneumonia being the main fatal complication.

In these children, it is necessary to be very careful when diagnosing Measles. To be sure, typical clinical signs, such as Koplik's spots and leukopenia, must be present, and therefore many 'morbilliform' skin rashes in newborns are reported as Measles, but the diagnosis is only false -positive [16]. Apparently, placental transmission occurs, but asymmetrically. Considering the reports of absence of disease in the neonate in the presence of maternal infection at the time of delivery, transplacental passage is not frequent. Intrauterine disease has been reported and can be serious. It is considered that the serological test has little relevance in congenital Measles, due to the immaturity of the immune system [12].

Perinatal measles, or early childhood measles, as it may also be called, has been reported since the 1940 s, possibly due to the general immunity of mothers in urbanized areas and the corresponding protection of their newborns by passive immunization through the placenta. There are publications that described a child in which the viral genome was detected up to 140 days after birth. It is believed that there is a relation in immunological immaturity at the time the child was infected. Measles contamination before 1 year of life is a major threat factor for subacute sclerosing panencephalitis, caused by the cerebral immaturity of the infected child [15].

This exclusively human virus is preserved in the population by a continuous chain of acute infections and can be extinguished by mass vaccination of the population. Measles infection generally has a favorable course, but there is a possibility of serious complications, especially when it manifests itself days before or after delivery, there is a risk of congenital Measles related to an incomplete transfer of protective maternal antibodies [15].

The clinical condition becomes quite variable in the newborn: skin rash, pneumonia, or even asymptomatic. There is also a greater chance of developing subacute early sclerosing panencephalitis with a fatal course, it is indicated the administration of immune serum soon after exposure. The diagnosis is made by classical serological test of peripheral blood and RT-PCR of saliva samples.

The use of injectable immunoglobulin is not recommended in pregnant women due to major side effects on immunity [16]. The only indication for an exposed pregnant woman is prophylaxis within 6 days after contact with a person infected with Measles, confirmed serologically. Although they do not contain published data on immunoglobulin prophylaxis in newborns specifically, its efficacy is well defined within 6 days and the use of the triple viral vaccine and the RT-PCR of samples allows a rapid and reliable diagnosis of Measles infection in newborns before the appearance of signs and symptoms, thus, allowing the use of polyvalent intravenous immunoglobulin [14], considering that IgM positivity isslow, being detected 3 days after the appearance of the rash [17].

\section{Conclusion}

It is concluded that Measles is considered rare in newborns, although it is possible to affect them in cases of susceptible mothers, and therefore further studies are needed in favor of clinical knowledge of the disease course in this age group. However, it is known that the Measles RT-PCR test in salivary samples can be used before the development of symptoms in the infant to confirm early transmission from mother to child and that the use of the viral RT-PCR test of samples has allowed a rapid and reliable diagnosis of measles infection in a newborn before the presence of clinical signs and late positivity of anti-measles IgM, therefore allowing the use of intravenous polyvalent immunoglobulin. Apparently, there is a higher incidence of prematurity in the presence of Gestational Measles and there is no relation among the disease and abortion. 


\section{Conflicts of Interest}

The authors declare that they have no conflicts of interest.

\section{Financing}

This research did not receive any specific subsidies from public, commercial or non-profit sectors.

\section{References}

1. Rodrigues TS, Santos LS, Lacerda GS, Kanaan S (2019) Diagnóstico clínico, laboratorial e profilático do sarampo no Brasil. J Bras Patol Med Lab 55(4).

2. Organização Pan-Americana de Saúde (OPAS) (2019) Folha informativa - Sarampo.

3. (2020) Sarampo.

4. Alerta Sarampo (2019) Orientações a Profissionais de Saúde.

5. Patzer JD, Martins LG, Ranieri T, et al. Boletim Epidemiológico. pp. 16.

6. Brasil (2017) Ministério da Saúde. Secretaria de Vigilância em Saúde Departamento de Vigilância Epidemiológica. Coordenação-geral de Desenvolvimento da Epidemiologia em Serviços. Guia de Vigilância Epidemiológica 2: 113-128.

7. Carvalho AL, Dolabela A, Meira A (2018) Alerta Sarampo. Sociedade mineira de pediatria. P. 8.

8. Drut R, Drut RM (1988) Measles Pneumonia in a Newborn. Pediatric Pathology.
9. Domingues CMAS, Pereira MCCQ, Santos ED, Siqueria MM, Ganter B (1997) A evolução do sarampo no Brasil e a situação atual. Inf Epidemiol Sus 6(1).

10. Almeida CMS, Souza LGD, Coelho GN, Almeida KC (2020) Correlação entre o aumento da incidência de sarampo e a diminuição da cobertura vacinal dos últimos 10 anos no Brasil. Braz J Hea Rev 3: 406-415.

11. Sousa JG, Barbosa HC, Silva MO, Campos JRE, Luz DCRP (2019) Movimentoantivacinação: uma ameaçaà humanidade. Rev e-ciência 7: 41-42.

12. Korones SB (1988) Uncommon vírus infections of the Mother, Fetus, and Newborn: Influenza, Mumps and Measles. Clin Perinatol 15: 259-272.

13. Kohn JL (1933) Measlesin new born infants (maternal infection). The Journal of Pediatrics 3: 176-180.

14. Giusti D, Burette J, Nguyen Y, Leveque N, Graesslin O, et al. (2013) Virological diagnosis and management of two cases of congenital measles. Journal of medical virology 85: 2136-2138.

15. Ragazzi B, Lopes S, Vaz-de-lima A, Rehder L, Paul R, et al. (2005) Congenital and neonatal measles during na epidemic in São Paulo, Brazil in 1997. The pediatric infectious disease journal 24: 377-378.

16. Barsegar B, Itofmann H, Zweymiller E (1972) The diagnosis of measles in the newborn and infant age groups. Z Kinderheilk 113: 175-184.

17. Ohji G, Satoh H, Satoh H, Mizutani K, Iwata K, et al. (2009) Congenital measles caused by transplacental infection. The pediatric infectious disease journal 28: 166-167. 